\title{
Chiral condensate thermal evolution at finite baryon chemical potential within chiral perturbation theory
}

\begin{abstract}
R. García Martín and J. R. Peláez
Departamento de Física Teórica II, Universidad Complutense de Madrid, 28040 Madrid, Spain (Received 29 August 2006; revised manuscript received 18 October 2006; published 8 November 2006)

We present a model independent study of the chiral condensate evolution in a hadronic gas, in terms of temperature and baryon chemical potential. The meson-meson interactions are described within chiral perturbation theory and the pion-nucleon interaction by means of heavy baryon chiral perturbation theory, both at one loop, and nucleon-nucleon interactions can be safely neglected within our hadronic gas domain of validity. Together with the virial expansion, this provides a systematic expansion at low temperatures and chemical potentials, which includes the physical quark masses. This can serve as a guideline for further studies on the lattice. We also obtain estimates of the critical line of temperature and chemical potential where the chiral condensate melts, which systematically lie somewhat higher than recent lattice calculations but are consistent with several hadronic models. We have also estimated uncertainties due to chiral parameters, heavier hadrons, and higher orders through unitarized chiral perturbation theory.
\end{abstract}

\section{INTRODUCTION}

One of the most interesting questions about QCD is its phase diagram, and, in particular, the transition from a hadron gas to a quark-gluon plasma, and the restoration of chiral symmetry at finite baryon density. On the experimental side, there are several experiments such as CERES, KEK, STAR, SPS, LHC, and RHIC that probe this transition at different values of temperature and baryon density. On the theoretical side, first principle calculations on the quark-gluon phase performed on the lattice have been traditionally hindered by the well-known fermion determinant sign problem. The search for alternative approaches to overcome this problem, at least for some values of the chemical potential, have recently bolstered the activity in lattice QCD at finite density (see for example [1-5] for a review and further references). However, lattice studies still present some difficulties in the infinite volume extrapolation and most importantly in that they do not use realistic values for the quark and hadron masses, particularly the pions and kaons, which are the lightest mesons and the most abundant at low temperatures.

However, from the hadronic phase it is also possible to obtain model independent predictions by means of chiral perturbation theory (ChPT) [6-8] which is the low energy effective theory of QCD (see [9] for introductions and reviews). Let us recall that the spontaneous chiral symmetry breaking of QCD requires the existence of eight massless Goldstone bosons that can be identified with the pions, kaons, and the eta. Therefore they are the most relevant degrees of freedom at low energies. With these fields, the ChPT Lagrangian is built as the most general derivative expansion, over $4 \pi f_{\pi} \simeq 1.2 \mathrm{GeV}$ (the symmetry breaking scale), respecting the symmetry constraints of QCD. Hence, ChPT is the low energy effective theory of QCD. Actually, there is also an explicit symmetry breaking due to the small quark masses that give rise to a small mass for the pions, kaons, and the eta, which are thus just pseudoGoldstone bosons. For this reason ChPT is an expansion also in masses which are treated perturbatively. The leading term is fixed once we know the symmetry breaking scale, and the next orders contain a finite number of constants that absorb the infinities generated from loops, rendering the calculations finite order by order. Baryons can also be included in the Lagrangian respecting chiral symmetry but their treatment is more involved due to their large masses. Within heavy baryon chiral perturbation theory (HBChPT) [10], this problem is overcome for the meson-baryon interaction by an additional expansion over the baryon mass. Since we are only interested in the quark condensate in a hadronic gas, we will neglect the nucleonnucleon interactions. In particular, this leaves cold nuclear matter outside our domain of applicability. We therefore have a model independent formalism derived from QCD that provides a systematic expansion at low energies and chemical potentials. This approach has proven very successful and works remarkably well within the meson sector, whereas for the meson-baryon sector the convergence is somewhat slower.

In order to include the thermodynamic effects of the temperature and chemical potential, we will use another model independent approach, namely, the virial expansion [11-13]. It is a simple and successful technique already applied to describe dilute gases made of interacting pions [14] and other hadrons [12]. In contrast to lattice approaches, it is very straightforward to introduce the baryon chemical potential. For most thermal observables it is enough to know the low energy scattering phase shifts of the particles within the gas, which could be taken from experiment, avoiding any model dependence. However, since we are interested in the quark condensate, defined as a derivative of the pressure with respect to the quark 
masses, one needs a model independent theoretical description since it cannot be obtained directly from experiment. Thus, in this work we extend to finite baryon chemical potential, $\mu_{B}$, the model independent approach that combines the virial expansion and ChPT, a method already applied at $\mu_{B}=0$ in [14-16].

For the condensate, it is therefore particularly important to note that we are using the physical hadron masses in all our calculations. Let us also remark that the expansion that relates hadron masses to quark masses, particularly for pions, shows some of the best convergence properties in ChPT, much better than the energy expansion itself. We therefore hope that our results at low energy and chemical potential could serve as a guideline for a correct inclusion of mass effects in further studies, for instance, on the lattice, at least in the isospin limit.

The plan of the work is as follows. In the next section we introduce in detail the virial formalism and make a rough estimation, using the free gas, of its applicability bounds in the temperature-chemical potential plane. With a brief introduction to ChPT and the hadron mass dependence on quark masses, we incorporate the interactions, which we do in two different scenarios. A pure SU(2) gas of pions and nucleons, where we include systematically contributions up to a given order, and a more realistic gas including kaons, etas, and heavier hadrons, where we neglect certain contributions due to the Boltzmann suppression. We will show the size of the different terms paying particular attention to the pion-nucleon interaction, and we will provide phenomenological parametrizations of the condensate melting temperature. In addition, we will estimate by extrapolation the melting line in the temperature-chemical potential plane. Finally, we will calculate our uncertainties due to the imperfect knowledge of chiral parameters and of the high energy behavior. The latter will be done by using unitarized chiral amplitudes. We will conclude with a summary and discussion of our results.

\section{THE VIRIAL EXPANSION}

The thermodynamics of a system of hadrons is encoded in the grand canonical potential density $z=\epsilon_{0}-P$, where we have explicitly extracted $\epsilon_{0}$, which is due to the existence of a vacuum expectation value even at $T=0$, and $P$ stands for the pressure [14,17]. In our case we are interested in a multicomponent relativistic gas made of pions, kaons, etas, and nucleons. Later on we will introduce heavier hadrons. In addition, we will assume that only the strong interactions are relevant and that the baryon density, defined as $n_{B}-n_{\bar{B}}$, is conserved. For that reason, the pressure will depend on the temperature $T$ and a baryon chemical potential $\mu_{B}$. Note that the nonstrange quark chemical potential $\mu_{q}=\mu_{B} / 3$ is also frequently used in the literature. The relativistic virial expansion [11-13] reads

$$
\beta P=\sum_{i}\left(B_{i}^{(1)} \xi_{i}+B_{i}^{(2)} \xi_{i}^{2}+\sum_{j \geq i} B_{i j}^{\mathrm{int}} \xi_{i} \xi_{j}+\cdots\right),
$$

where $\xi_{i}=\exp \left[\beta\left(\mu_{i}-M_{i}\right)\right], \beta=1 / T, M_{i}$ is the mass of the $i$ species and $\mu_{i}=0, \pm \mu_{B}$ for mesons, baryons (antibaryons), respectively. The coefficients

$$
B_{i}^{(n)}=\frac{g_{i} \eta_{i}^{n+1}}{2 \pi^{2}} \int_{0}^{\infty} d p p^{2} e^{-n \beta\left(\sqrt{p^{2}+M_{i}^{2}}-M_{i}\right)}
$$

correspond simply to the virial expansion of the pressure for a free gas

$$
\begin{aligned}
\beta P_{\text {free }}= & -\sum_{i} \frac{g_{i} \eta_{i}}{2 \pi^{2}} \int_{0}^{\infty} d p p^{2} \\
& \times \log \left[1-\eta_{i} e^{-\beta\left(\sqrt{p^{2}+M_{i}^{2}}-\mu_{i}\right)}\right],
\end{aligned}
$$

where $\eta_{i}=1$ for bosons and $\eta_{i}=-1$ for fermions. The interactions appear in the virial expansion through the $S$-matrix $[13,14]$. For the meson-meson and pion-nucleon interactions relevant for this work, this can be recast in terms of the elastic scattering phase shifts, thus we can write

$$
B_{i j}^{\mathrm{int}}=\frac{e^{\beta\left(M_{i}+M_{j}\right)}}{2 \pi^{3}} \int_{M_{i}+M_{j}}^{\infty} d E E^{2} K_{1}(\beta E) \Delta^{i j}(E),
$$

where $K_{1}(x)$ is the first modified Bessel function of the second kind and

$$
\Delta^{i j}=\sum_{I, J, S}(2 I+1)(2 J+1) \delta_{I J S}^{i j}(E),
$$

$\delta_{I, J, S}^{i j}$ being the $i j \rightarrow i j$ phase shifts (defined so that $\delta=0$ at threshold) of the elastic scattering of a state $i j$ with welldefined isospin, total angular momentum, and strangeness $I, J, S$. For the nucleon-nucleon interaction, using the $S$-matrix representation could be more convenient than the phase shifts, but here we are only interested in a sufficiently diluted hadron gas, so that we can neglect $N N$, and the above formalism is enough for our purposes. Thus, in this work we will use the virial expansion together with ChPT phase shifts, extending previous works to include a nonvanishing baryon chemical potential in a hadron gas. We will study the validity of this approach in a section below.

Let us now recall that the quark masses appear in the Lagrangian as $m_{q} \bar{q} q$, therefore the nonstrange quark condensate is given by [14]

$$
\langle\bar{q} q\rangle_{T, \mu_{B}}=\frac{\partial z}{\partial \hat{m}}=\langle 0|\bar{q} q| 0\rangle-\frac{\partial P}{\partial \hat{m}} .
$$

Note that we are working in the isospin limit, using a common mass $\hat{m}=\left(m_{u}+m_{d}\right) / 2$ both for the $u$ and $d$ quarks, and $\langle 0|\bar{q} q| 0\rangle \equiv\langle 0|\bar{u} u+\bar{d} d| 0\rangle$. The $\langle 0|\bar{s} s| 0\rangle$ condensate, which is smaller, and whose thermal evolution is slower than the nonstrange one [16] could also be studied 
with similar methods, but this lies beyond our present scope.

\section{CHIRAL PERTURBATION THEORY}

Let us then briefly explain our use of ChPT and fix some notation, starting from the purely mesonic sector. Since the ChPT Lagrangian is built as the most general derivative and mass expansion [6-9], the meson-meson interaction amplitudes are obtained as a series in even powers of momenta and masses, both denoted generically $p^{2}$. The leading order starts at $O\left(p^{2}\right)$ and is universal, only depending on one scale $F$, which at leading order can be identified with the pion decay constant $f_{\pi}$. At next to leading order (NLO), the amplitudes contain one-loop diagrams with $O\left(p^{2}\right)$ vertices plus the tree level contributions from the $O\left(p^{4}\right)$ Lagrangian. Indeed, all one-loop calculations can be renormalized in terms of a set of $O\left(p^{4}\right)$ parameters, $L_{k}(\mu)$ and $H_{k}(\mu), \mu$ being the renormalization scale, which can be determined from a few experiments, or in the case of $H_{2}^{r}$, from the resonance saturation hypothesis [18], and used for further predictions at low temperatures. In Table I we list the values of these parameters that we will use for the amplitudes in this work.

Within ChPT, partial waves $t_{I J S}$ are obtained as an expansion in even powers of momenta and masses. Dropping for simplicity the $I J S$ indices, we find $t(s)=$ $t_{2}(s)+t_{4}(s)+\cdots$, where $t_{n}(s)=O\left(p^{n}\right)$. Since we need elastic amplitudes for the virial expansion, the phase shift $\delta_{I J S}(s)$ is simply the complex phase of its corresponding partial wave. In principle, the ChPT series is only valid at low energies compared with $4 \pi f_{\pi} \simeq 1 \mathrm{GeV}$, although in practice it is limited to momenta of the order of 200$300 \mathrm{MeV}$ above threshold. For this reason, we cannot apply our expansions for temperatures beyond that range. We will study the applicability of our approach with more detail in the next section.

Baryons have a mass of the order of the chiral expansion scale $4 \pi f_{\pi}$, but they can also be included as degrees of freedom in a chiral effective Lagrangian if they are treated

TABLE I. One-loop ChPT low energy constants used for our calculations. Those in the ChPT column are taken from $[7,19]$, whereas those in the IAM column are taken from [20]. $H_{2}^{r}\left(M_{\rho}\right)$ is taken from [18] for both cases.

\begin{tabular}{lcc}
\hline \hline & ChPT & \multicolumn{1}{c}{ IAM } \\
\hline$L_{1}^{r}\left(M_{\rho}\right)$ & $0.4 \pm 0.3$ & $0.561 \pm 0.008$ \\
$L_{2}^{r}\left(M_{\rho}\right)$ & $1.35 \pm 0.3$ & $1.21 \pm 0.001$ \\
$L_{3}$ & $-3.5 \pm 1.1$ & $-2.79 \pm 0.02$ \\
$L_{4}^{r}\left(M_{\rho}\right)$ & $-0.3 \pm 0.5$ & $-0.36 \pm 0.02$ \\
$L_{5}^{r}\left(M_{\rho}\right)$ & $1.4 \pm 0.5$ & $1.4 \pm 0.02$ \\
$L_{6}^{r}\left(M_{\rho}\right)$ & $-0.2 \pm 0.3$ & $0.07 \pm 0.03$ \\
$L_{7}$ & $-0.4 \pm 0.2$ & $-0.44 \pm 0.003$ \\
$L_{8}^{r}\left(M_{\rho}\right)$ & $0.9 \pm 0.3$ & $0.78 \pm 0.02$ \\
$H_{2}^{r}\left(M_{\rho}\right)$ & $-3.4 \pm 1.1$ & $-3.4 \pm 1.1$ \\
\hline \hline
\end{tabular}

as heavy particles in a covariant framework called HBChPT [10]. In this case, the series is organized in terms of order $N=1,2,3 \ldots$ that contain powers of $p^{N} /\left(F^{2 l} M_{N}^{N+1-2 l}\right)$, where $l=1, \ldots,(N+1) / 2$. Once again, the divergences at each order are absorbed in a set of parameters, which in the notation of [21] are called $a$ 's at $O\left(p^{2}\right)$ and $b$ 's at $O\left(p^{3}\right)$. We provide in Table II the values we have used, but we want to remark that these parameters are strongly correlated and other sets can be found in [23].

The HBChPT framework is especially well suited for our approach, not only since $\pi N$ scattering has been calculated to one loop, but also because it has been unitarized $[22,24]$ describing the $\Delta(1232)$ resonance correctly, which will be of use for the last section. In particular, we will use the third order calculation [21,23]. Note that there is a fourth order calculation available [25], that has also been unitarized [22,24], but it introduces many more chiral parameters (up to 18 parameters in total) with very large uncertainties, and just a slight improvement over the third order results. Let us also remark that the second order coefficients, which are called $a_{i}$ in the formalism of [21] that we follow here, can be translated to other coefficients often used in the literature, called $c_{i}$, which are not dimensionless. The values listed in the HBChPT column in Table II correspond to

$$
\begin{array}{ll}
c_{1}=-1.06 \pm 0.06 & c_{2}=3.4 \pm 0.5 \\
c_{3}=-5.74 \pm 0.15 & c_{4}=3.7 \pm 0.2
\end{array}
$$

in $\mathrm{GeV}^{-1}$ units. These are perfectly compatible with the recent values given in [26]

$$
\begin{array}{ll}
c_{1}=-0.9_{-0.2}^{+0.5} & c_{2}=3.3 \pm 0.2 \\
c_{3}=-4.7_{-1.0}^{+1.2} & c_{4}=3.5_{-0.2}^{+0.5} .
\end{array}
$$

The corresponding values used for unitarized $\mathrm{HBChPT}$ in the last section are

TABLE II. Low energy constants for $O\left(p^{3}\right)$ HBChPT used in our calculations, for the nonunitarized and unitarized cases (taken from [22]).

\begin{tabular}{lcc}
\hline \hline & HBChPT & HBChPT + IAM \\
\hline$a_{1}$ & $-2.60 \pm 0.03$ & $-1.36 \pm 0.02$ \\
$a_{2}$ & $1.40 \pm 0.05$ & $0.438 \pm 0.015$ \\
$a_{3}$ & $-1.00 \pm 0.06$ & $-0.70 \pm 0.04$ \\
$a_{5}$ & $3.30 \pm 0.05$ & $1.29 \pm 0.04$ \\
$\tilde{b}_{1}+\tilde{b}_{2}$ & $2.40 \pm 0.3$ & $3.06 \pm 0.3$ \\
$\tilde{b}_{3}$ & $-2.8 \pm 0.6$ & $-0.41 \pm 0.27$ \\
$\tilde{b}_{6}$ & $1.4 \pm 0.3$ & $-1.5 \pm 0.2$ \\
$b_{16}-b_{15}$ & $6.1 \pm 0.6$ & $7.4 \pm 0.5$ \\
$b_{19}$ & $-2.4 \pm 0.4$ & $-3.7 \pm 0.2$ \\
\hline \hline
\end{tabular}




$$
\begin{aligned}
& c_{1}=-0.75 \pm 0.02 \quad c_{2}=1.4 \pm 0.5 \\
& c_{3}=-3.1 \pm 0.3 \quad c_{4}=1.5 \pm 0.2 .
\end{aligned}
$$

As the last point concerning ChPT, and as we advanced in the Introduction, the chiral condensate virial calculation requires the knowledge of the quark mass dependence. Within ChPT, since we deal with hadrons, we actually recast Eq. (6) as follows [14]:

$$
\begin{gathered}
\langle\bar{q} q\rangle=\langle 0|\bar{q} q| 0\rangle-\sum_{h} \frac{\partial M_{h}}{\partial \hat{m}} \frac{\partial P}{\partial M_{h}} \\
=\langle 0|\bar{q} q| 0\rangle\left(1+\sum_{h} \frac{c_{h}}{2 M_{h} F^{2}} \frac{\partial P}{\partial M_{h}}\right),
\end{gathered}
$$

where, for further convenience we have introduced the constant $F$, which is the pion decay constant in the chiral limit, and we have defined the coefficients

$$
c_{h}=-F^{2} \frac{\partial M_{h}^{2}}{\partial \hat{m}}\langle 0|\bar{q} q| 0\rangle^{-1},
$$

which encode the hadron mass dependence on the quark mass. The $c_{\pi}$ coefficient in SU(2) ChPT was calculated in [14]. Here we will use the SU(3) ChPT expressions at $O\left(M_{\pi}^{4}\right)$ which, for $h=\pi, K, \eta$ can be read in [16]. Numerically they amount to

$$
c_{\pi}=0.9_{-0.4}^{+0.2}, \quad c_{K}=0.5_{-0.7}^{+0.4}, \quad c_{\eta}=0.4_{-0.7}^{+0.5} .
$$

Using the $O\left(M_{\pi}^{4}\right)$ calculation of the nucleon mass within HBChPT [23,27], we find

$$
c_{N}=3.6_{-1.9}^{+1.5}
$$

where we have used the recent values [26] for the HBChPT parameters in Eq. (8). This corresponds to a nucleon sigma term:

$$
\sigma_{\pi N}=40_{-21}^{+17} \mathrm{MeV}
$$

where the uncertainties are estimated assuming uncorrelated errors and provide a very conservative range. Had we used the constants in Eq. (7), we would have found $51_{-23}^{+13} \mathrm{MeV}$. We have checked that the difference between these two central values will amount to roughly $1 \mathrm{MeV}$ or less in our melting temperatures within the validity region of the approach, that we will study in the next section.

Finally, let us analyze the hierarchy of the different terms. First of all, we see that the contributions to the virial expansion of the pressure are exponentially suppressed as $\exp \left(-\beta M_{h}\right)$. Nevertheless, the $\exp \left(\beta \mu_{B}\right)$ factor for the nucleons can overcome the previous thermal suppression if the chemical potential is of the same order as their mass. These exponentials are inherited by the derivatives. In addition, we observe that $c_{\pi} / M_{\pi}>c_{N} / M_{N} \gg c_{K} / M_{K}>$ $c_{\eta} / M_{\eta}$. For the above two reasons, pions and the interactions of the other species with pions are the dominant contributions for studying the melting of the condensate.
Nucleons become comparable only when their chemical potential is of the order of their mass. In addition, since nucleon-nucleon interactions are much stronger at low energies than those of pion-pion or pion-nucleon, we will carefully exclude the region with too high nucleon density.

As an illustration, and in order to compare with other results in the literature as well as to obtain an estimate on the reliability of the virial expansion, we will first study briefly the case of a free gas.

\section{THE FREE GAS AND THE DOMAIN OF VALIDITY OF OUR APPROACH}

The interest of this case is that we have a closed expression for the pressure, already given in Eq. (2), which can be integrated numerically and compared with its second order virial expansion, namely, Eq. (16) with all $B_{i j}^{\mathrm{int}}=$ 0, i.e.,

$$
\beta P \simeq \sum_{h} B_{h}^{(1)} \xi_{h}+B_{h}^{(2)} \xi_{h}^{2}
$$

First of all, in Fig. 1 we have plotted the relative abundances of the most relevant species as a function of the temperature, which we parametrize in terms of their density

$$
n_{h}\left(\mu_{B}, T\right)=\frac{g_{h}}{2 \pi^{2}} \int_{0}^{\infty} d p \frac{p^{2}}{e^{\beta\left(\sqrt{p^{2}+M_{h}^{2}}-\mu_{h}\right)}-\eta_{h}} .
$$

We see that the lightest particles, the pions, are the main component of the hadronic gas up to temperatures as high as $200 \mathrm{MeV}$. As previously remarked, we can also notice that nucleons form a small fraction of the gas unless we

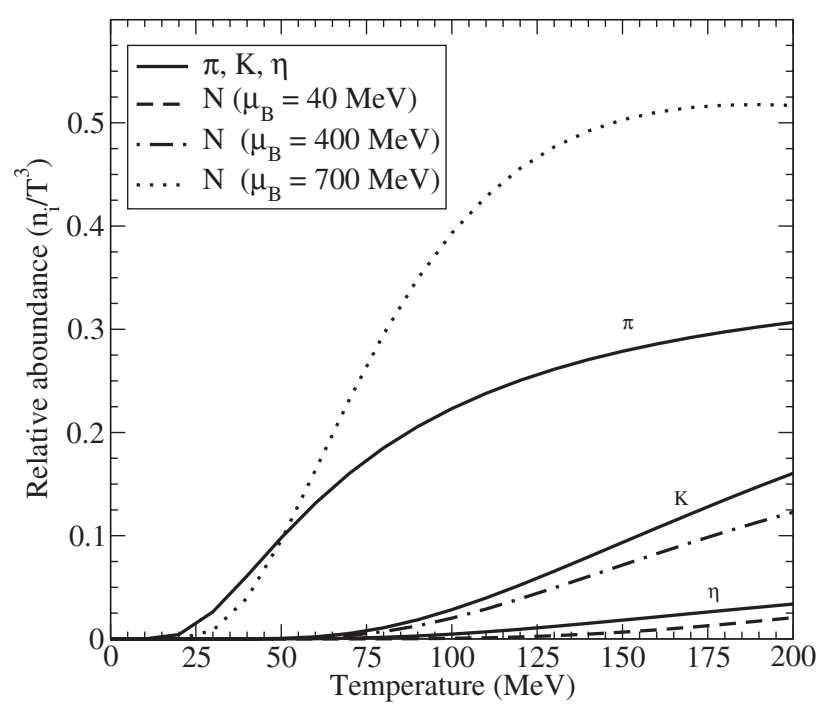

FIG. 1. Density over $T^{3}$ of different species in a free hadronic gas. The pion population is by far the largest up to $200 \mathrm{MeV}$, except at very high baryon chemical potentials, where nucleons dominate. 


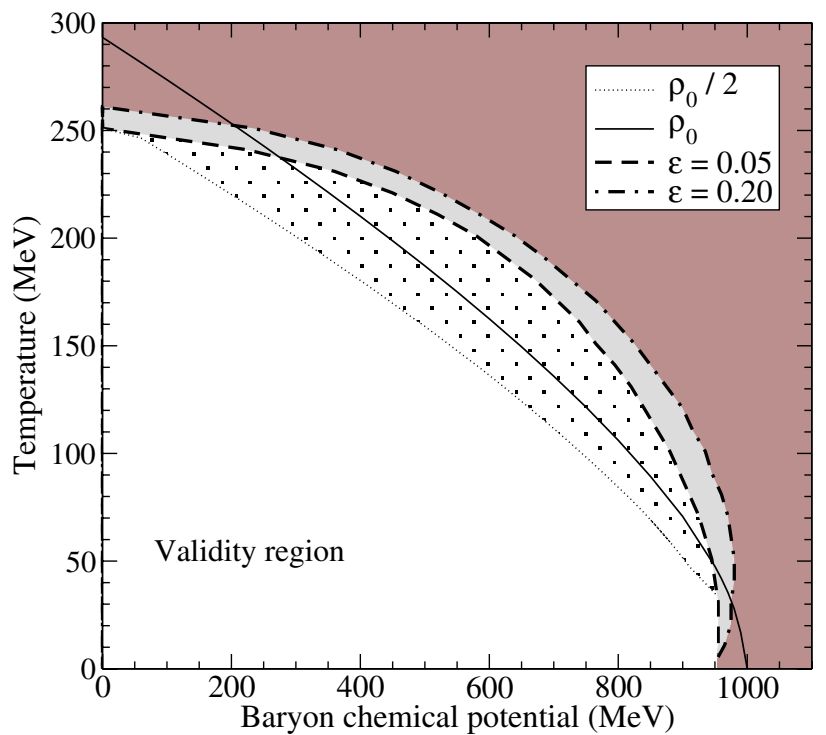

FIG. 2 (color online). Estimation of the region where the virial expansion may provide a good approximation to the quark condensate. Within the region inside the dashed line, and for a free gas of pions and nucleons, the relative error $\epsilon$ of the second order virial expansion with respect to the analytic expression is less than 5\%. The virial approximation deteriorates rapidly beyond that "validity region." As commented in the text, we also require the nucleon density to be smaller than the saturation density $\rho_{0}$ (the thick continuous line) and therefore we exclude the dotted area. The remaining white area is our approximate validity region.

reach high chemical potentials, of the order of $500 \mathrm{MeV}$ or higher.

Next, in Fig. 2 we show, in the $\left(\mu_{B}, T\right)$ plane, the relative error between the exact calculation using Eq. (2) and the second order virial expansion for the chiral condensate, Eq. (16). This we define as

$$
\epsilon=\frac{\left|\langle\bar{q} q\rangle_{\text {exact }}-\langle\bar{q} q\rangle_{\text {virial }}\right|}{\frac{1}{2}\left|\langle\bar{q} q\rangle_{\text {exact }}+\langle\bar{q} q\rangle_{\text {virial }}\right|}
$$

We see that the second order virial expansion provides a fairly good approximation $\epsilon \simeq 0.05$ for moderate temperatures and chemical potentials (roughly $T<200 \mathrm{MeV}$ and $\mu_{B}<800 \mathrm{MeV}$ ) and slightly beyond we obtain just qualitatively correct results $(\epsilon \simeq 0.20)$, since the expansion deteriorates rather rapidly. In addition, and since our scope is just to describe a simple hadronic gas, the nuclear matter regime should be excluded from the validity region. In fact, nuclear matter, even at low temperatures, is not a gas but a fluid [17], since, at low momenta, $N N$ interactions are an order of magnitude larger than $\pi \pi$ or $\pi N$ interactions. Just for illustration, we have plotted in Fig. 2 as a continuous line, the points where the saturation density, $\rho_{0} \simeq$ $0.16 \mathrm{fm}^{-3}$, is reached.

Let us however remark that we are not interested in all the hadron thermodynamics, but just in a particular quan- tity, the quark condensate, that does not depend on the interactions themselves but on their derivative with respect to quark masses. Thus, the enhancement due to $N N$ interactions with respect to $\pi N$ or $\pi \pi$ is not as large for the quark condensate as it may be for other thermodynamic quantities that depend on the $N N$ interactions. In particular, it has been shown [28], using an equation of state for the nuclear matter regime based on a chiral Lagrangian, that the evolution of the quark condensate with nuclear density is well reproduced up to somewhat beyond $\rho_{0} / 2$ just with a linear term proportional to the nuclear $\pi N$ sigma term, but that higher orders in density are relevant beyond (see Fig. 3 in [28]). Since the nucleon-nucleon interaction lies outside the scope of this work, but the $\pi N$ interaction is included, we only consider densities below $\rho_{0} / 2$. Note that with this choice we lie on the safe side. In addition, the uncertainties due to the $\pi N$ interaction, that will be studied later in this work, are much bigger than the neglected effects of higher orders in density up to densities much larger than $\rho_{0} / 2$.

In summary, in Fig. 2, the dotted area stands for densities above $\rho_{0} / 2$ and the remaining white area in Fig. 2 will be referred as the "validity region," in the understanding that this is just a very crude estimate. Fortunately, we will see that, once we introduce the interactions, most of the interesting phenomena, including our extrapolations for the condensate melting, occur within the bounds of this validity region, so that the virial expansion is reasonably under control and definitely not diverging wildly. In particular, the region of relevance to study the freeze-out or the phase

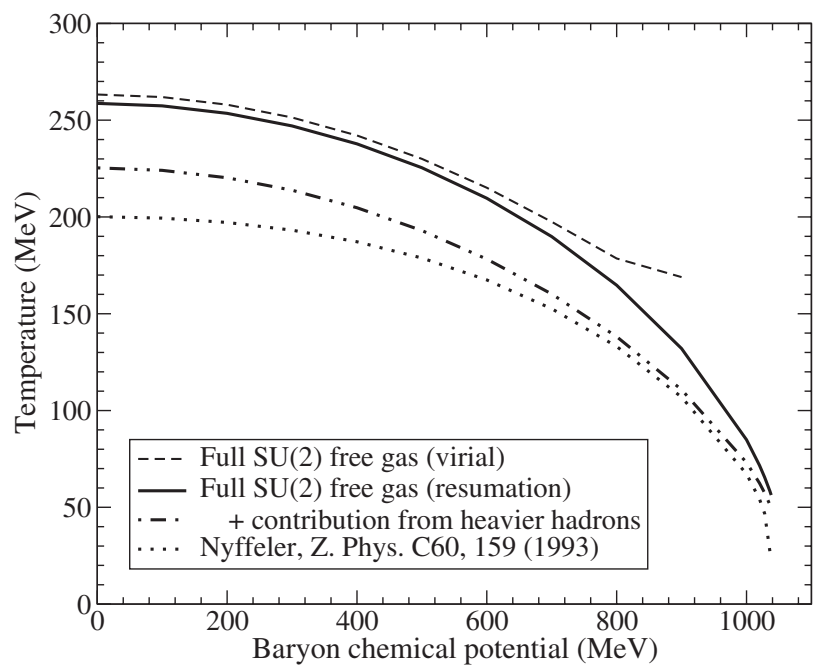

FIG. 3. Condensate melting line in the $\left(\mu_{B}, T\right)$ plane for a free pion and nucleon gas (continuous line). Note that it is very well approximated by the extrapolated line from the second order virial expansion (dashed) as long as $\mu_{B}<800 \mathrm{MeV}$. The melting is faster when adding free heavier hadrons (dash-dotted line). Finally, we compare with the free gas results using crude estimates of $\partial M_{h} / \partial m_{q}$ based on the number of valence quarks [30] (dotted line). 
transition in relativistic heavy ion collisions, $\mu_{B}=$ 40-50 MeV, $T \simeq 170-200 \mathrm{MeV}$ [29] lies within our domain of validity.

Finally, we show in Fig. 3 the condensate melting line in the $\left(\mu_{B}, T\right)$ plane using either the closed form of the grand canonical potential density or the one extrapolated from our second order virial expansion. First of all, if we only consider $\pi, K, \eta$, and nucleons, we note that, starting from zero chemical potential, the second order virial melting line indeed follows closely the complete calculation with Eq. (2), although it deviates abruptly around $T \simeq 180 \mathrm{MeV}$ and $\mu_{B}=800 \mathrm{MeV}$, that nevertheless lies beyond the "validity region."

In Fig. 3 we also show the melting line that results if we add the contribution of the heavier hadrons in the free gas approximation by using

$$
\begin{aligned}
\Delta\langle\bar{q} q\rangle & =-\sum_{h} \frac{\partial \Delta P}{\partial M_{h}} \frac{\partial M_{h}}{\partial \hat{m}} \\
& =\frac{1}{2 \pi^{2}} \sum_{h} g_{h} M_{h} \frac{\partial M_{h}}{\partial \hat{m}} \int_{0}^{\infty} d p \frac{p^{2} / \epsilon_{h}(p)}{e^{\beta\left(\epsilon_{h}(p)-\mu_{h}\right)}-\eta_{h}},
\end{aligned}
$$

with $\epsilon_{h}(p)=\sqrt{p^{2}+M_{h}^{2}}$.

In such a case, the extrapolated melting line falls slightly outside the estimated validity region. We will see in the next sections that, by adding interactions, an important part of the melting line moves within the validity region.

Figure 3 is also relevant because it allows us to compare with previous results existing in the literature that use the free hadron gas, Eq. (3), to study the condensate, including also heavier particles as above. In particular, we can compare with the melting lines obtained in [30,31]. We can observe that there is a quantitative difference between those calculations and the one we present here. Such a difference is mainly due to the dependence of the hadron masses on quark masses $\partial M_{h} / \partial \hat{m}$, which in those works was not obtained from ChPT, but simply estimated as $\partial M_{h} / \partial \hat{m} \simeq N_{q}$ (that is the number of light quarks, except for the pion that was chosen to be 10) [30] and $\partial M_{h} / \partial M_{\pi}^{2} \simeq A / M_{h}$ with $A \sim 0.9-1.2$ [31]. Table III shows the comparison between the $c_{h}$ coefficients obtained from these estimations vs the ChPT or HBChPT $O\left(p^{4}\right)$ calculations in Eqs. (13) and (14), which we consider very

TABLE III. Coefficients $c_{h}$ as calculated from estimates in other hadronic models [30,31] versus the analytic values obtained in ChPT or HBChPT.

\begin{tabular}{lccc}
\hline \hline$c_{h}$ & ChPT/HBChPT & Nyffeler & Tawfik-Toublan \\
\hline$c_{\pi}$ & $0.9_{-0.4}^{+0.2}$ & 2.0 & $0.8-1.1$ \\
$c_{K}$ & $0.5_{-0.7}^{+0.4}$ & 0.7 & $1.6-2.1$ \\
$c_{\eta}$ & $0.4_{-0.7}^{+0.5}$ & 0.8 & $2.4-3.2$ \\
$c_{N}$ & $3.6_{-1.9}^{+1.5}$ & 4.2 & $0.6-0.7$ \\
\hline \hline
\end{tabular}

accurate, since the $M_{\pi}^{2} /\left(4 \pi f_{\pi}\right)^{2}$ ChPT expansion converges very well. Let us remark that the $c_{h}$ coefficients in those works are generically larger than ours for $h=\pi, K$, and $\eta$. Hence, the effect of using such estimates is to accelerate the thermal melting of the condensate. In particular, in Figure 3, we show the results of [30], giving rise to a much lower critical temperature. This explains why the free gas results in $[30,31]$ yield melting temperatures much below those obtained in a free gas using ChPT calculations for $\partial M_{h} / \partial \hat{m}$, as in $[14,15]$. As a matter of fact, we have checked that our approach reproduces the results in [30] just by changing the $c_{h}$ coefficients.

In what follows, and given the fact that the free and interacting contributions are separated in the virial expansion, we show the results both expanding the free gas part to second order in the virial expansion, and also using the analytic expression.

\section{THE INTERACTING SU(2) GAS OF PIONS AND NUCLEONS}

In the literature the $\mathrm{SU}(2)$ flavor case $[2,3,14,15]$ is frequently studied, by considering the strange quark as heavy. This is simpler, since the pions are the only pseudo-Goldstone bosons and there is a clearer suppression of heavier hadrons due to their heavy masses. On top of that $\mathrm{SU}(2) \mathrm{ChPT}$ and HBChPT show a much better convergence than their $\mathrm{SU}(3)$ counterparts. In addition, we can use the one-loop calculations within HBChPT of the $\pi N$ scattering amplitudes.

Thus, we will consider the second order virial expansion of a gas of pions and nucleons, where pions interact among themselves and with nucleons. The nucleon-nucleon contribution to the condensate melting is suppressed since $c_{\pi} / M_{\pi}>c_{N} / M_{N}$ and also because of the Boltzmann suppression of the nucleon population, which can only be compensated for baryon chemical potentials which lie outside the validity region of the virial expansion and are therefore beyond our scope.

The $\mu_{B}=0$ case within ChPT was first studied in [14] both with the virial expansion and by an effective field theory calculation of the grand canonical potential density. Later on, the ChPT virial study was extended to a finite pion chemical potential in [15]. In both works, the only interacting particles were the pions, and all other hadrons were added in the free approximation.

In this section, apart from the one-loop ChPT $\pi \pi$ scattering amplitudes, we have included the $O\left(p^{3}\right)$ calculation of $\pi N$ within HBChPT [21,23]. First we show in Fig. 4 the results at $\mu_{B}=0$, and we can notice how the free $\mathrm{SU}(2)$ gas melting temperature lies beyond the virial validity region, but the pion-pion interaction brings it down to $\sim 230 \mathrm{MeV}$, within the naive validity region at $\mu_{B}=0$ and in agreement with [14-16]. When we further introduce free nucleons, we get an additional decrease down to $224 \mathrm{MeV}$. Of course, within our approach, the melting 


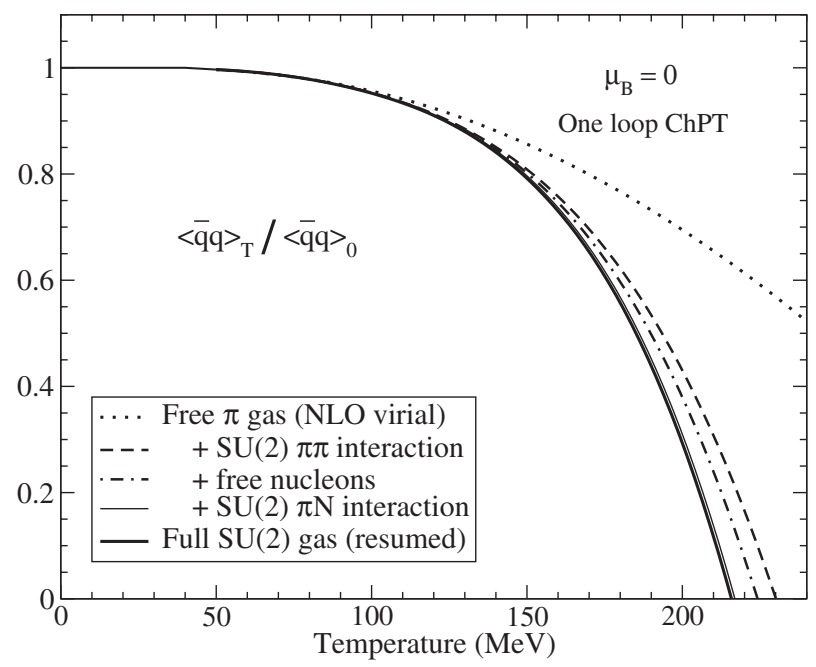

FIG. 4. Chiral condensate thermal evolution at zero baryon chemical potential for the SU(2) pion and nucleon gas. The free gas extrapolated melting temperature falls entirely beyond the validity region of the virial expansion, but $\pi \pi$ interactions bring it below $230 \mathrm{MeV}$. Although $\mu_{B}=0$, both the free nucleons and their interaction with pions accelerate sizably the condensate melting. Note that, when interactions are introduced, the difference between calculating the free gas contributions exactly (resumed) or with the virial expansion is negligible, and the two curves fall on top of each other.

temperatures are just extrapolations and lie near the edge of the validity region. What is not an extrapolation is the behavior at low $T$, where the different contributions are calculated consistently with the virial and chiral expansions. However, their effect is more difficult to see in the figures, and we quote the extrapolated melting temperatures because it is easier to quantify the relative size of the different contributions. One should always keep in mind that these are just extrapolations.

Surprisingly, the $\pi N$ interaction, which is the new contribution that we are adding, has a sizable effect even at $\mu_{B}=0$, decreasing the melting temperature by another $7 \mathrm{MeV}$, down to $217 \mathrm{MeV}$.

Let us emphasize that each additional contribution decreases the melting temperature further, but since the melting of the condensate accelerates near the melting point (the curve becomes steeper as it gets close to zero), even if the new contribution has a similar size as the previous one, its effect on the melting temperature seems smaller. That is the reason why the relatively large effect of $\pi N$ is even more surprising. A nice illustration of this effect is seen in Fig. 4 since the total result is practically the same no matter whether we use the virial expansion or the analytic (resumed) expression for the free terms. Indeed, both curves fall on top of each other in the figure. This is in contrast with Fig. 3, where there was a difference of roughly $5 \mathrm{MeV}$ observed at $\mu_{B}=0$ between the free gas melting temperature depending on whether we used the analytic form of the grand canonical potential density or its second order virial expansion. The reason is that, in Fig. 3, after adding all interactions, the slope of the condensate is so steep that there is almost no difference in the melting temperature, $1 \mathrm{MeV}$, between using the virial expansion for the free terms or not.

Let us now set $\mu_{B} \neq 0$. In Fig. 5 we show in the $\left(\mu_{B}, T\right)$ plane the melting line of the free $S U(2)$ gas of pions and nucleons, the gas with only interacting pions, and, finally, the effect of adding the $\pi N$ interaction. The effect of the latter becomes bigger as $\mu_{B}$ increases from zero, and becomes maximum around $\mu_{B}=600-700 \mathrm{MeV}$, where it produces a decrease in the melting temperature of about $40 \mathrm{MeV}$ with respect to the gas without $\pi N$ interactions. Up to a chemical potential of $\mu_{B}=40-50 \mathrm{MeV}$, the region of relevance [29] for relativistic heavy ion collisions, the decrease in the melting temperature amounts roughly to $10 \mathrm{MeV}$ when we include the $\pi N$ interaction.

In Fig. 6 we present a comparison of our pure SU(2) results with lattice estimates in the literature $[2,32]$. Note that our melting temperature for a given $\mu_{B}$ lies systematically above the corresponding one on the lattice. This could already be noticed in the $\mu_{B}=0$ case [14], both with the analytic ChPT calculation of the partition function and the virial expansion $[14,15]$.

To facilitate future comparison of our results with other calculations, we have performed a simple quadratic fit to our central results up to $500 \mathrm{MeV}$, giving the following phenomenological expression for the dependence of the melting temperature with the baryon chemical potential:

$$
\frac{T_{c}\left(\mu_{B}\right)}{T_{c}(0)}=1-0.03155(2)\left(\frac{\mu_{B}}{T_{c}(0)}\right)^{2},
$$

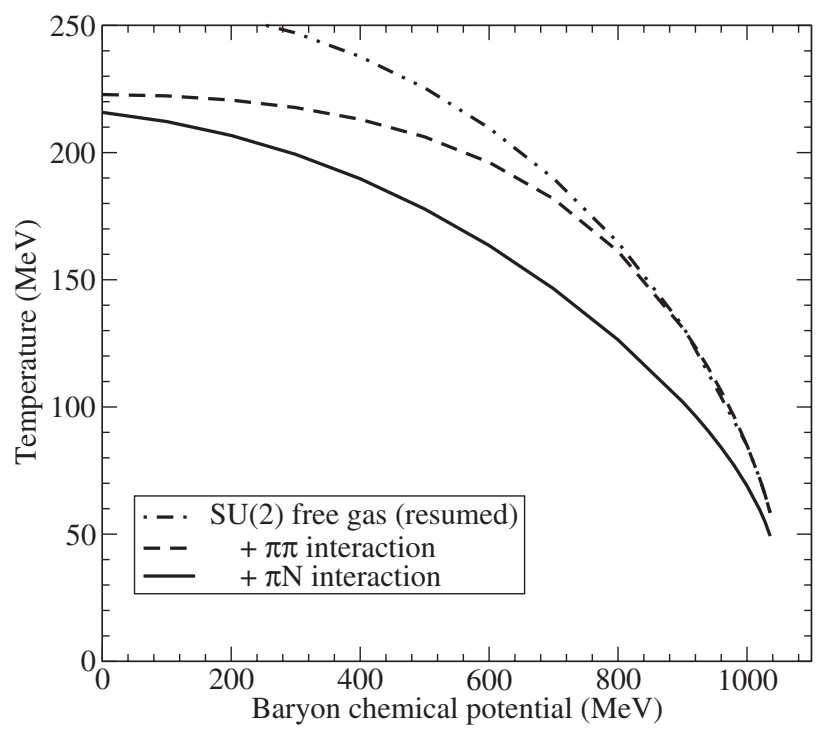

FIG. 5. Melting line in the $\left(\mu_{B}, T\right)$ plane of the chiral condensate for an $\mathrm{SU}(2)$ gas of hadrons. We show the result for the free gas, but also adding the $\pi \pi \mathrm{ChPT} \mathrm{SU}(2)$ interaction to one loop, and the line resulting from adding the $\pi N$ interaction to third order in HBChPT. 
R. GARCÍA MARTÍN AND J.R. PELÁEZ

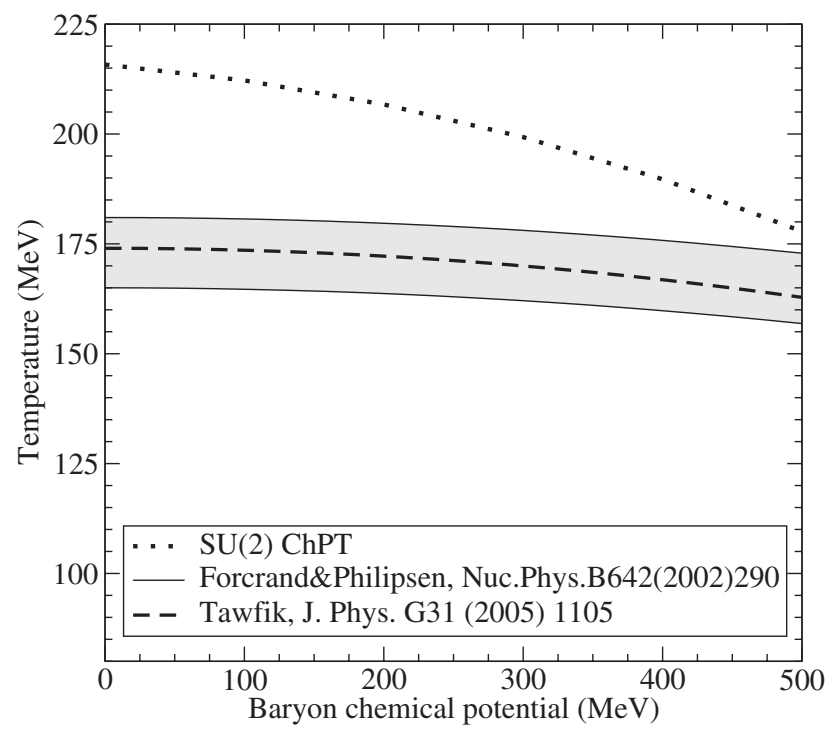

FIG. 6. Comparison between our results and other estimates in the literature. The gray band corresponds to the fit in [2], while the dashed line corresponds to the one shown in [32]. Note that these works obtain values for $T_{c}$ that are systematically lower than our model independent approach (dotted line).

where the uncertainty is due only to the statistical fit to the central value, and does not contain the uncertainties in our approach, mostly due to the chiral parameters, which will be treated in a separated section below.

\section{REALISTIC HADRON GAS}

In the previous section we considered a gas made just of pions and nonstrange nucleons. However, in a real gas, we should consider all hadrons. This we will do by including them as free particles. The only exception will be the kaons and etas, that, in view of Fig. 1, are sufficiently abundant up to $200 \mathrm{MeV}$ to deserve a separate treatment and include their interaction with a pion. All other interactions are severely suppressed by Boltzmann and $c_{h} / M_{h}$ factors. At very large $\mu_{B}$, the heavier nucleons may not be Boltzmann suppressed, but that is beyond our estimated validity range.

Thus, in Fig. 7, we compare the condensate thermal evolution at $\mu_{B}=0$ of the SU(2) pion and nucleon gas including $\pi \pi$ and $\pi N$ interactions (thick continuous line) with the results obtained adding further contributions that are numerically sizable. First, we consider the effect of free kaons and etas, which slightly accelerate the vanishing of the condensate, decreasing the extrapolated melting temperature by $\sim 4 \mathrm{MeV}$. Next, we have included the kaon and eta loops in the $\pi \pi$ interaction. This amounts to calculating $\pi \pi$ scattering in SU(3) ChPT instead of SU(2). Since the $\pi \pi$ interaction is by far the dominant one, this one-loop effect decreases the melting temperature by $\sim 2.5 \mathrm{MeV}$. When we include the one-loop interactions of $\pi K$ and $\pi \eta$, we observe a further decrease of $\sim 6.5 \mathrm{MeV}$. We have checked that, if we remove the nucleons, these results are in agreement with [16].
PHYSICAL REVIEW D 74, 096003 (2006)

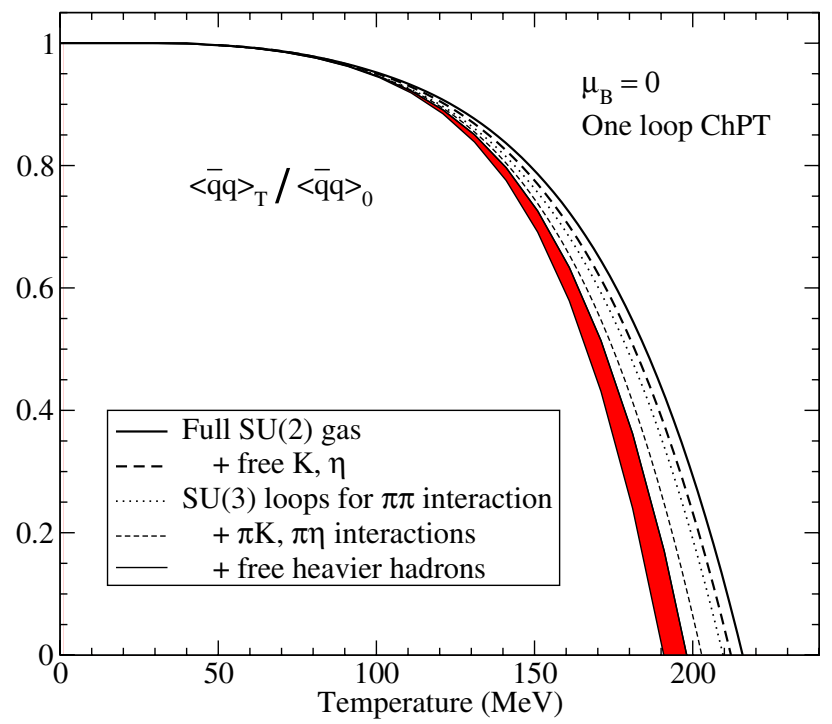

FIG. 7 (color online). Condensate thermal evolution at $\mu_{B}=0$ in a gas of hadrons. Starting from the SU(2) gas including $\pi \pi$ and $\pi N$ interactions, we show the effect of adding kaons and etas, both as real and virtual states in $\pi \pi$ loops, as well as the effect of the $\pi K$ and $\pi \eta$ ChPT one-loop interactions. The band includes the estimated uncertainties due to free heavier hadrons.

To end with the $\mu_{B}=0$ case, we add the heavier hadrons, where we have estimated that

$$
\frac{\partial M_{h}}{\partial \hat{m}} \simeq \alpha N_{u, d}^{h}, \quad \text { (heavier hadrons) }
$$

where $\alpha$ is an adimensional constant and $N_{u, d}^{h}$ is the number of valence $u$ or $d$ quarks in the hadron. For nucleons, following [33], and from our Eqs. (13) and (14), we estimate $\alpha_{N} \simeq 1.9_{-1.0}^{+0.7}$. For the other heavier hadrons, we have estimated $\alpha \simeq 0.5-2.5$, which roughly cov-

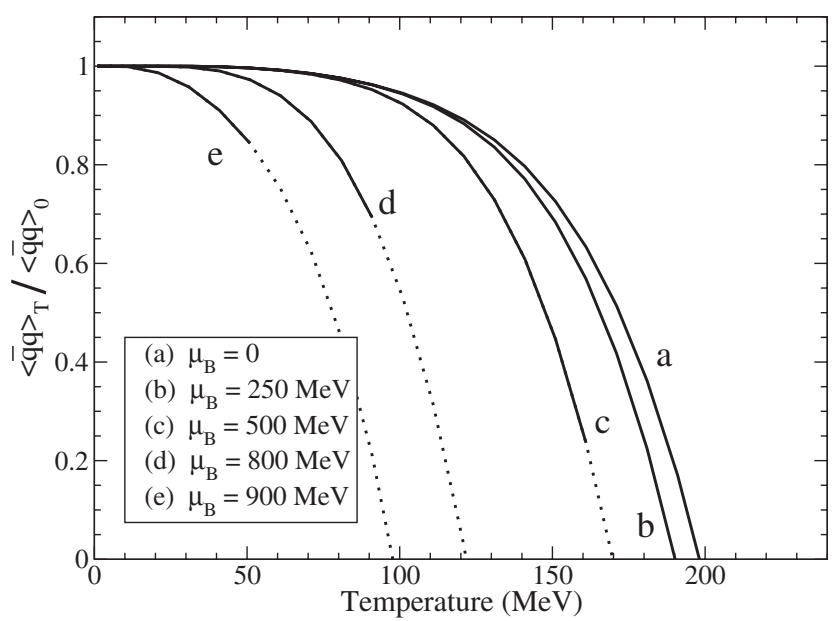

FIG. 8. Condensate thermal evolution in a gas of hadrons for different constant values of $\mu_{B}$. The dotted pieces of the curves lie beyond the "validity region" estimated in the text. Note the lines just correspond to central values. 
ers the range of $\alpha$ obtained for the nucleons and light mesons.

Let us now turn to $\mu_{B} \neq 0$. In Fig. 8 we show curves for the evolution of the chiral condensate for different constant values of the chemical potential. We can see how the increase of $\mu_{B}$ accelerates the melting of the condensate, and that the effect of the chemical potential on the melting temperature is less than $10 \mathrm{MeV}$ until $\mu_{B}$ is of order $250 \mathrm{MeV}$. In addition, we have plotted the curves as continuous lines within the "validity region" estimated in previous sections and as dotted lines outside. In this way we see that, when $\mu_{B}$ is of the order of the nucleon mass, the approach deteriorates rapidly, although it can still give reasonable results at very low temperatures. A different perspective is provided in Fig. 9, where we now show the condensate evolution versus $\mu_{B}$ for constant $T$. Note that, as we get close to $T=0$, the evolution with the chemical potential is almost a constant, the condensate in vacuum, within the validity region. At exactly $T=0$ all fugacities vanish, except those of nucleons when $\mu_{B}>$ $M_{N}$, but that lies beyond the validity region and cannot be studied with our approach.

Finally, in Fig. 10, we present the melting lines in the $\left(T, \mu_{B}\right)$ plane, showing first the complete $\mathrm{SU}(2)$ pion and nucleon gas result, to which we have added all the effects of kaons and etas. As it could be expected, the biggest difference is observed at low $\mu_{B}$. In particular, in the interesting $\mu_{B} \simeq 40-50 \mathrm{MeV}$ region [29] we find that there is a $\sim 10 \mathrm{MeV}$ additional decrease due to kaons and etas, on top of the one we already found in the previous section due to the $\pi N$ interaction. For higher energies the effect of $K$ and $\eta$ contributions are less evident, not because they decrease, but because they become relatively less impor-

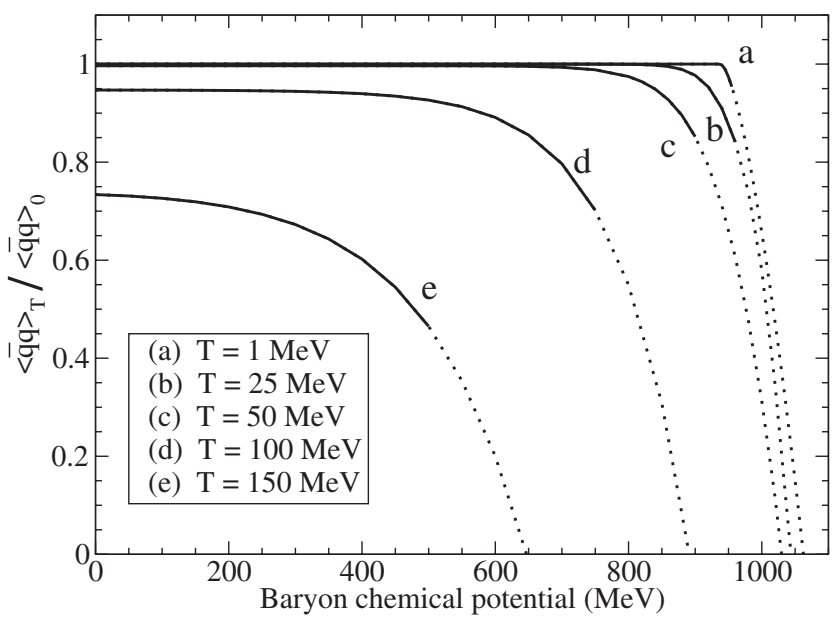

FIG. 9. Condensate evolution with the baryon chemical potential in a gas of hadrons for different constant values of $T$. The dotted pieces of the curves lie beyond the "validity region" estimated in the text. Note the lines just correspond to central values.

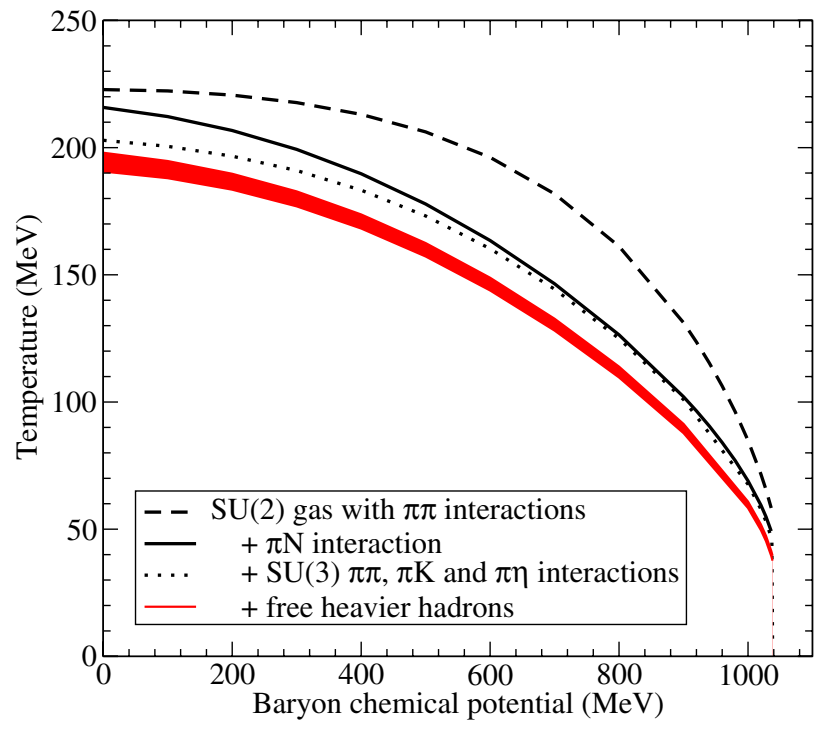

FIG. 10 (color online). Our final result for the chiral condensate melting line in the $\left(\mu_{B}, T\right)$ plane. Starting from the $\mathrm{SU}(2)$ gas with $\pi \pi$ interactions, we show the effect of $\pi N$ interactions and of adding kaons and etas. The dark area covers the uncertainty due to heavier hadrons estimated as explained in the text.

tant with respect to the nucleon free terms and the larger $\pi N$ interaction.

In the last section we will discuss the comparison of our results with some previous works in the literature, but first we will study the uncertainties within our approach.

\section{UNCERTAINTIES DUE TO THE CHIRAL PARAMETERS}

In previous sections we have shown results for the central values of the chiral parameters given in Table I and II. In this section we show the uncertainty due to the errors in the chiral parameters also listed in those tables by adding in quadrature the uncertainties caused by each one of the parameters independently. Let us remark that the dependence on these parameters is twofold. On the one hand, they appear in the interactions through the phase shifts in Eq. (5), but, on the other hand, some of them also appear in the $c_{h}$ coefficients in Eq. (12) that parametrize the hadron mass dependence on the quark masses [16]. Indeed, the errors in Eqs. (13) and (14) correspond to the uncertainties in the chiral parameters.

Thus, in Fig. 11 we show again as a dark band our final extrapolated melting line in the $\left(\mu_{B}, T\right)$ plane, which includes the uncertainties due to heavier hadrons. The region between the dashed lines covers also the uncertainties due to the chiral parameters. The error is highly asymmetric since, as we have already commented, it becomes more and more difficult to decrease the melting temperature by adding new effects, so that the inner dashed line is much closer to our "central" results.

We also remark that the uncertainties grow very fast at higher $\mu_{B}$ values in good agreement with the estimated 


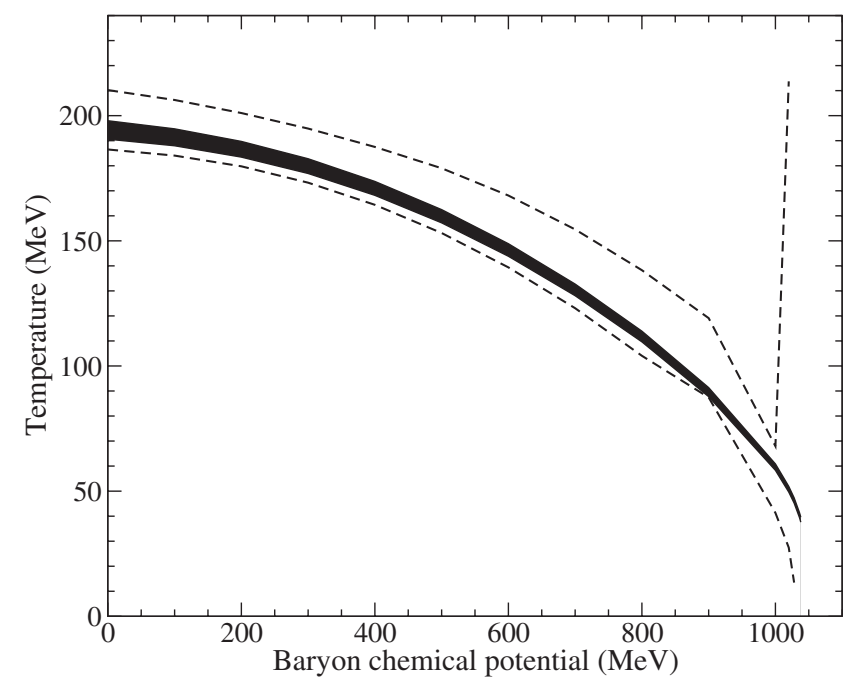

FIG. 11. Our final result for the chiral condensate melting line in the $\left(\mu_{B}, T\right)$ plane. The dark area covers the uncertainties due to the heavier hadrons, which we have included as free components of the gas. The area between dashed lines corresponds to the uncertainty in the parameters of ChPT and HBChPT listed in Table I and II.

range of validity of the chiral expansion. At sufficiently large values of $\mu_{B}$, our uncertainties are too large even for qualitative predictions. However, for small or moderate chemical potentials, say $\mu_{B} \ll M_{N}$, we get quite stable predictions, and the extrapolated melting line is determined up to a 10 to $15 \mathrm{MeV}$ uncertainty.

In Fig. 12 we compare our melting line estimates with previous works. We show both our full error band (between the dotted lines) and that due to heavier hadrons only (light

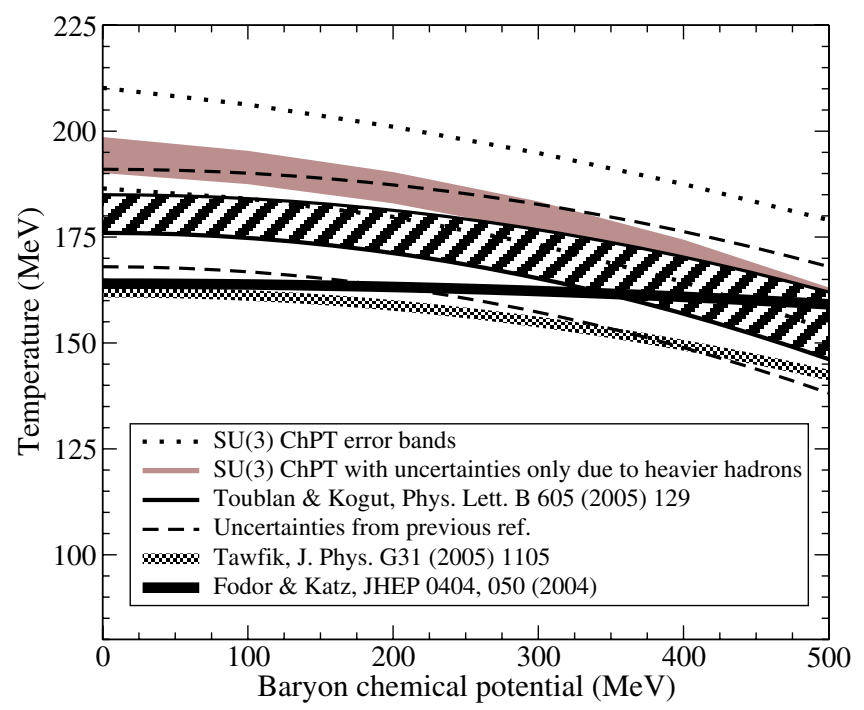

FIG. 12 (color online). Comparison between our results and others in the literature. Our melting temperatures are consistent within errors with Ref. [34] but systematically higher than $[4,32]$. gray band). Note that we are consistent within uncertainties with the hadronic model in [34], but, systematically, our melting temperatures are somewhat higher than estimates using lattice [4,32]. Note that, as expected, and in contrast with lattice results, our melting temperatures always occur a few $\mathrm{MeV}$ above the chemical freeze-out temperature that has been estimated from $p_{\perp}$ spectra at RHIC as $T_{f}=$ $165 \pm 7 \mathrm{MeV}, \mu_{B}^{f}=41 \pm 5 \mathrm{MeV}[29]$.

Finally, to ease future comparisons with our results, we provide a simple quadratic fit to our results up to $500 \mathrm{MeV}$, giving the following phenomenological expression for the dependence of the melting temperature with the baryon chemical potential:

$$
\frac{T_{c}\left(\mu_{B}\right)}{T_{c}(0)}=1-0.025_{-0.011}^{+0.017}\left(\frac{\mu_{B}}{T_{c}(0)}\right)^{2} .
$$

In contrast with the fit given in the pure $\mathrm{SU}(2)$ case, Eq. (20), the errors now are much larger since they take into account the uncertainty due to the chiral parameters and other heavier hadrons.

\section{UNITARIZED INTERACTIONS}

Up to this point, we have been using a model independent approach. To that end we have used chiral perturbation theory, which is a low momenta expansion, to describe the interactions between hadrons. However, in the virial coefficients, Eq. (4), the momentum integral extends to infinity, and one may wonder if the extrapolation of ChPT amplitudes to high energies beyond their applicability range may be distorting the results. In order to check the importance of the high energy contribution to those integrals, we will thus extend the ChPT amplitudes by means of unitarization, and, in particular, the inverse amplitude method (IAM), which provides a remarkably good description of the meson-meson scattering data up to much higher energies. This will introduce a mild model dependency but will allow us to estimate how large the effect of the high energy extrapolation is in the virial integrals.

Let us first briefly review unitarization and the IAM. For a detailed description of the method and its results, we refer to [20,35-37]. Note that, in view of Eqs. (4) and (5), we need phase shifts of elastic amplitudes of definite isospin $I$ and angular momentum $J$. Such amplitudes are called partial waves, $t_{I J}(s)$, although for brevity we will drop the $I, J$ indices. Unitarity for elastic partial waves implies that

$$
\operatorname{Im} t(s)=\sigma(s)|t(s)|^{2}, \quad \sigma(s) \equiv 2 q_{\mathrm{CM}} / \sqrt{s},
$$

where $q_{\mathrm{CM}}$ is the center of mass momentum. Note that this implies that the modulus of the partial wave in the elastic regime is bounded by $|t(s)|<\sigma^{-1}(s)$.

The ChPT partial waves are obtained as an expansion in momenta and masses $t(s)=t_{2}(s)+t_{4}(s)+\cdots$, where $t_{2}=O\left(p^{2}\right), t_{4}=O\left(p^{4}\right)$, where $p$ denotes a generic mo- 
menta or pseudo-Goldstone boson mass. Thus, unitarity is only satisfied order by order:

$$
\operatorname{Im} t_{2}(s)=0, \quad \operatorname{Im} t_{4}(s)=\sigma(s)\left|t_{2}(s)\right|^{2},
$$

This means that, since the truncated ChPT series grow like polynomials, unitarity can be badly violated at high energies. In addition, typical features of strong interactions, which are the resonances that saturate the above unitarity bound, are absent from the ChPT partial waves, since they correspond to poles in the second Riemann sheet that cannot be described with polynomials. In practice, pure NLO ChPT only provides a description of meson-meson scattering data up to energies of the order of $200 \mathrm{MeV}$ above threshold.

However, partial wave unitarity, Eq. (23), fixes completely the imaginary part of the inverse amplitude since it follows that $\operatorname{Im} t^{-1}=-\sigma(s)$. Consequently, in the elastic regime a unitary partial wave can be written as

$$
t(s)=\frac{1}{\operatorname{Re} t^{-1}(s)-i \sigma(s)},
$$

and therefore we only need an approximation to $\operatorname{Re} t^{-1}(s)$. The inverse amplitude method (IAM) simply approximates $\operatorname{Re} t^{-1}(s)$ by the NLO ChPT result to find

$$
t^{\mathrm{IAM}}(s)=\frac{t_{2}^{2}}{t_{2}-\operatorname{Re}_{4}-i \sigma t_{2}^{2}}=\frac{t_{2}(s)}{1-t_{4}(s) / t_{2}(s)},
$$

where in the last step we have used Eq. (24). In this way we satisfy unitarity while respecting the NLO chiral expansion, which is recovered at low energies. In addition, the IAM can generate poles associated to resonances. The above derivation has been done for elastic amplitudes and yields amplitudes that indeed extend the ChPT calculations describing remarkably well the $\pi \pi$ and $\pi K$ scattering data up to roughly the first relevant inelastic threshold [35].

The formalism can be easily extended to the coupled channel case [20,36,37]. Let us illustrate the case when there are two coupled channels 1 and 2 [that could correspond, for instance, to $|1\rangle=\left|(\pi \pi)_{I J}\right\rangle$ and $\left.|2\rangle=\left|(\bar{K} K)_{I J}\right\rangle\right]$. We would then have four partial waves for the different choices of initial and final states $t_{i j}$, with $i, j=1,2$. Thus, the unitarity condition has now the following matrix form:

$$
\begin{gathered}
\operatorname{Im} T=T \Sigma T^{*}, \quad T=\left(\begin{array}{ll}
t_{11} & t_{12} \\
t_{21} & t_{22}
\end{array}\right), \\
\Sigma=\left(\begin{array}{cc}
\sigma_{1} & 0 \\
0 & \sigma_{2}
\end{array}\right) .
\end{gathered}
$$

Following a similar argument as for the one channel case above, we find

$$
T^{U}=T_{2}\left(T_{2}-T_{4}\right)^{-1} T_{2} .
$$

This expression has been shown to provide a very good description of meson-meson scattering up to $\sqrt{s} \simeq$
$1.2 \mathrm{GeV}$, much beyond the applicability range of standard ChPT. Note that this has been achieved with chiral parameters $L_{i}$ compatible with those of ChPT and therefore with a simultaneous description of the low energy and resonant regions [20,37]. In particular, the $f_{0}(600), \kappa(900), \rho(770)$, $K^{*}(892), f_{0}(980)$, and $a_{0}(980)$ masses and widths are well described together with their associated poles in the second Riemann sheet [37].

Concerning the pion-nucleon interactions, the unitarization could follow a similar line [24], but it can be improved [22] taking into account that HBChPT has a double expansion: the low momenta chiral expansion, that counts powers of $1 / f_{\pi}$ and a heavy fermion expansion in powers of $1 / M_{N}$, where $M_{N}$ is the nucleon mass. Factorizing explicitly the powers of $M_{N}$ and $f_{\pi}$, the expansion now reads

$$
\begin{aligned}
t= & \frac{M_{\pi}}{f_{\pi}^{2}} t^{(1,1)}+\frac{M_{\pi}^{2}}{f_{\pi}^{2} M_{N}} t^{(1,2)}+\frac{M_{\pi}^{3}}{f_{\pi}^{4}} t^{(3,3)}+\frac{M_{\pi}^{3}}{f_{\pi}^{2} M_{N}^{2}} t^{(1,3)} \\
& +\cdots,
\end{aligned}
$$

$t^{(n, m)}$ being dimensionless functions of $\omega / M_{\pi}$, where $\omega$ is the pion energy. Note that, contrary to the meson-meson case, odd orders of momenta do occur in the expansion.

The partial wave unitarity condition with one fermion in the final state simply reads: $\operatorname{Im} t=q_{\mathrm{CM}}|t|^{2}$, so that now

$$
t(s)=\frac{1}{\operatorname{Re} t^{-1}(s)-i q_{\mathrm{CM}}} .
$$

Once again [24] we can use the HBChPT series at NLO to approximate $\operatorname{Re}^{-1}(s)$. However, the HBChPT series converges much worse than in the meson-meson sector, and for that reason it is convenient to reorder the heavy baryon expansion (see Ref. [22]), to get the following unitarized amplitude:

$$
t^{\mathrm{IAM}} \simeq \frac{1}{\frac{f_{\pi}^{2}}{M_{\pi}}\left[t^{(1,1)}+\frac{M_{\pi}}{M_{N}} t^{(1,2)}+\frac{M_{\pi}^{2}}{M_{N}^{2}} t^{(1,3)}\right]^{-1}-M_{\pi} \frac{t^{(3,3)}}{\left(t^{(1,1)}\right)^{2}}} .
$$

It has been shown that this expression greatly improves the plain HBChPT description, extending it at least to the nearest relevant inelastic threshold, and, in particular, up to $\sqrt{s} \simeq 1400 \mathrm{MeV}$ in the $\Delta(1232)$ resonance channel, which is the most relevant feature of $\pi N$ scattering at low energies. Both the mass and width of this resonance are well described by the unitarized amplitude, together with its associated pole in the second Riemann sheet.

We will now use Eq. (28) for meson-meson and (31) for $\pi N$ scattering, in order to extend ChPT at high energies and check the contribution of the high momenta region in the virial integrals. The chiral constants we used for the unitarized amplitudes are also listed in Tables I and II. Note that for meson-meson scattering they are compatible with the values used in standard ChPT at NLO. The HBChPT parameters are not as well determined as those in the 
meson-meson sector, but it is important to note that the unitarization results are obtained with chiral parameters of natural size and quite compatible with different determinations from plain HBChPT.

At this point we want to remark that, since we are generating heavier resonances when using the unitarized amplitudes, we do not have to introduce them again as free particles in the gas, as we did in the previous section, to avoid double counting. In particular, we do not add now the $f_{0}(600), \kappa(900), \rho(770), K^{*}(892), f_{0}(980), a_{0}(980)$, and $\Delta(1232)$ resonances as free particles using Eq. (20), since they are generated by the IAM.

One advantage of unitarization is that some of the resonances are now included with their actual width (that is, interacting with the mesons), which is an effect that we could not take into account by including them as free components of the gas. A priori, this could be relevant, since these resonances have strong decays, i.e. large widths, and considering them stable is one of the greatest over-simplifications in hadronic models. We are thus quantifying also that effect in our previous model independent parametrizations. Furthermore, we had a large uncertainty in their $c_{h}$ or $\alpha_{h}$ parameters, which is now largely reduced since they appear in the meson-meson or meson-nucleon virial terms, whose $c_{h}$ coefficients, Eqs. (13) and (14), are much better determined.

Thus, in Fig. 13, we compare the condensate thermal evolution at $\mu_{B}=0$ that we obtained in previous sections with the results using the unitarized interactions. The dark narrow band covers, for the IAM results, the uncertainty due to heavier hadrons for the nonunitarized results. As commented in the previous paragraph, the fact that unitarized interactions include the effect of the first heavier

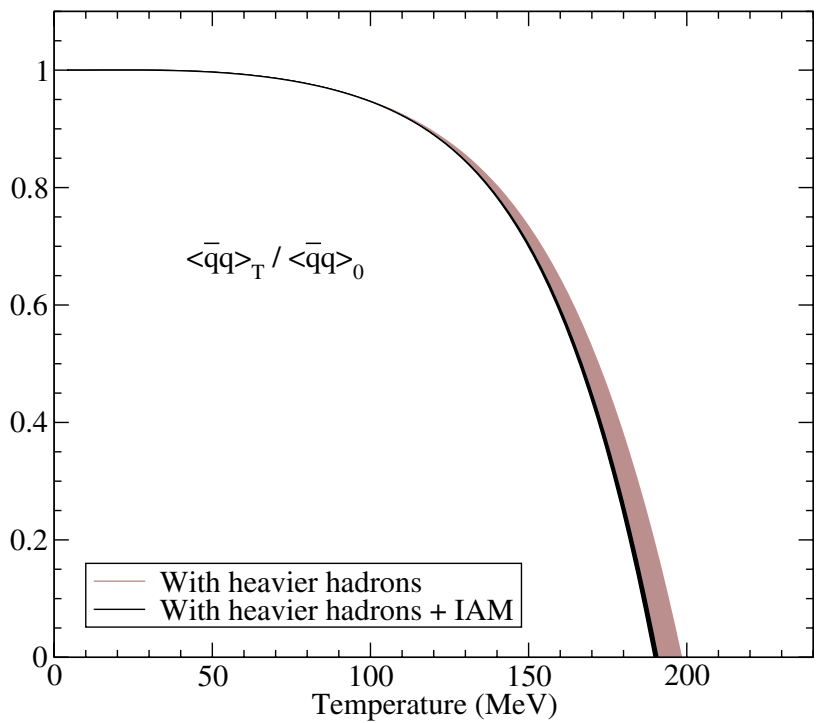

FIG. 13 (color online). Condensate thermal evolution at $\mu_{B}=$ 0 with ChPT unitarized interactions. The resulting melting temperature is slightly lower but very close to the model independent nonunitarized case within uncertainties. resonances accelerates the melting. In addition, since in the unitarized case what we call "heavier hadrons" are now the rest of hadrons, which are heavier and even less abundant, their associated uncertainty is much smaller. That is the reason why the dark band from the IAM is much narrower than the light band. Let us, however, note that, once the uncertainties are included, the unitarized and nonunitarized results are very close, showing a remarkable stability of our results at $\mu_{B}=0$.

Similar considerations hold for the uncertainty bands in Fig. 14. Again, the light band stands for the nonunitarized standard ChPT, and the much narrower dark band covers the uncertainty due to heavier hadrons of the IAM result. In this case, we see that the effect of unitarizing is larger, and the melting is systematically accelerated at moderate $\mu_{B} \neq 0$. The bigger difference is now due to the fact that, as pointed out above, the HBChPT series converges much worse than the meson-meson one, and the effect of unitarization is more dramatic, particularly for the $\Delta(1232)$ that lies very close to threshold. Still these results give support to our statement that our melting temperature estimates should be considered as upper bounds, although the differences between unitarized and nonunitarized are of about $10 \mathrm{MeV}$ at most.

Figure 15 shows the errors over this last band due to the uncertainties in the chiral parameters. The error is smaller than for the nonunitarized case. It should also be noted that both unitarized and nonunitarized cases overlap within their errors up to $\mu_{B} \simeq 300 \mathrm{MeV}$ and beyond that are never further than 2 standard deviations away.

Once again, the following quadratic fit provides a fairly good representation of our unitarized results up to

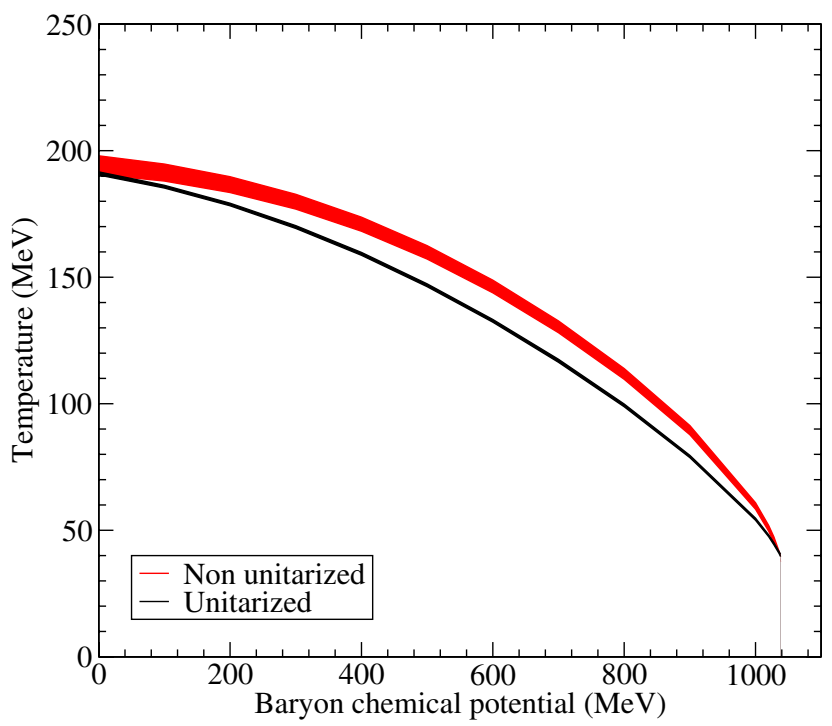

FIG. 14 (color online). Condensate melting lines in the $\left(\mu_{B}, T\right)$ plane with unitarized ChPT interactions compared to the nonunitarized ChPT. The unitarized case melts faster than the nonunitarized, due to the dynamically generated resonances that have their correct widths. 


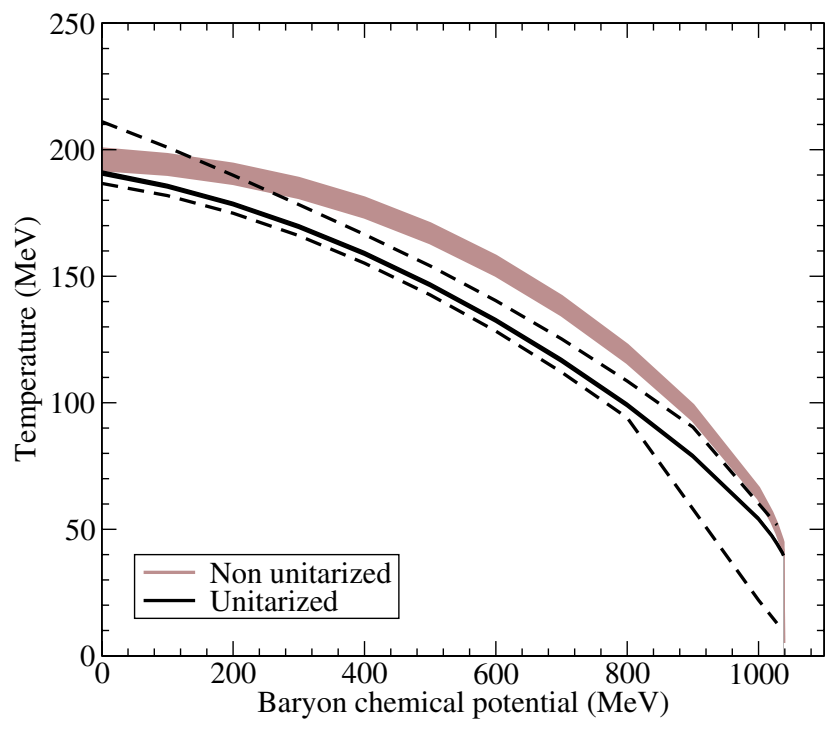

FIG. 15 (color online). The chiral condensate melting line in the $\left(\mu_{B}, T\right)$ plane for unitarized ChPT interactions. Note that the uncertainties due to the heavier hadrons (dark area) are much smaller than for the nonunitarized case in Fig. 11. The area between dashed lines corresponds to the uncertainty in the parameters of unitarized ChPT and HBChPT listed in Table I and II, and is also smaller than for the nonunitarized case.

$500 \mathrm{MeV}$ :

$$
\frac{T_{c}\left(\mu_{B}\right)}{T_{c}(0)}=1-0.032_{-0.001}^{+0.011}\left(\frac{\mu_{B}}{T_{c}(0)}\right)^{2},
$$

where, once more, the errors take into account the uncertainty due to both the chiral parameters and the heavier hadrons.

\section{SUMMARY AND DISCUSSION}

In this work we have presented a model independent description of a hadronic gas, based on the virial expansion and ChPT to NLO for the meson-meson interactions and third order for pion-nucleon within HBChPT. In particular, we have studied the melting of the chiral condensate $\langle\bar{q} q\rangle$ as a function of temperature, $T$, and baryon chemical potential, $\mu_{B}$. We have studied first the validity region, where the second order virial expansion is expected to provide a reasonably good description of the condensate evolution and nucleon density is low enough so that we can neglect $N N$ interaction. Since the virial expansion is a low density expansion and ChPT is a low energy effective theory, our approach is best applicable at low temperature and chemical potential, although it still seems to provide a fairly good description of the system up to temperatures of the order over $T=200 \mathrm{MeV}$ at $\mu_{B}=0$ and up to roughly $\mu_{B} \leq 900 \mathrm{MeV}$ at very low $T$ (see Fig. 2). This allows us to quantify the size of different contributions to the evolution of the condensate in terms of its extrapolated melting temperature and chemical potential. This is very likely a good estimate of the transition from "normal" hadronic matter, where chiral symmetry is spontaneously broken, to a phase where a chiral symmetry restoration may occur.

We use the virial expansion because it allows us to easily include both the chemical potential and the interactions in the thermodynamics of the system. The chemical potential is very hard to implement on the lattice, to study rigorously QCD, and our results may give some guidance on this respect. The interactions are included from the zero temperature and density elastic phase shifts, which are fairly well known from experiment. As a matter of fact, for most thermodynamical quantities, one could simply take the phase shifts from data. However, the calculation of the condensate requires the knowledge of the dependence on the quark masses, which is not given by experiment. Hence, a theory is needed to describe those data, and since perturbative QCD itself does not describe the low energy regime, one must turn to $\mathrm{ChPT}$, which is the low energy effective theory of QCD, for a model independent description.

Our use of ChPT is very relevant since it provides model independent and very reliable calculations of the hadrons mass dependence on quark masses, which is a critical quantity to follow the condensate evolution. This dependence has been frequently estimated roughly in the literature, but the ChPT calculation provides an expansion in terms of meson masses which shows a good convergence for pions, kaons, etas, and nucleons, which are the dominant components of the hadron gas. Furthermore, we have shown that several results obtained from free hadron gases in the literature can be reconciled with the interacting gas predictions if one uses these correct dependencies.

Next, we have presented results for an SU(2) gas including only pions and nucleons. This is of interest because of its simplicity and because we can include systematically all the effects up to a given order. This is in contrast with what we have called a realistic gas, where we have considered the interactions of kaons and etas with pions within the SU(3) ChPT formalism; although, formally, the $K K, \eta \eta$, and $\eta K$ interactions are of the same order, we have neglected them due to, first, the thermal suppression of kaons and etas compared with the pions and, second, the weaker dependence of their mass with respect to nonstrange quark mass.

In this work we have explicitly shown the size of different contributions to the condensate melting for $\mu_{B} \neq 0$. In particular, we have evaluated the free kaon, eta, and nucleon contributions, kaon and eta virtual effects in $\pi \pi$ interactions, as well as the $\pi K, \pi \eta$ interactions, these have also been calculated at $\mu_{B}=0$ without nucleons to check with previous results in the literature. In addition, we have included the $\pi N$ interaction, showing that it does have a sizable effect even at $\mu_{B}=0$. Around $\mu_{B}=$ 40-50 MeV the pion-nucleon interaction itself decreases the extrapolated melting temperature by $10 \mathrm{MeV}$. At higher baryon chemical potentials, the effect is larger. 
It is also relevant to remark that all contributions $d e$ crease the extrapolated melting temperatures. Intuitively, adding more states increases the possibilities of generating disorder, i.e., entropy, and accelerates the melting of the condensate. The interactions seem to reinforce this effect. Therefore, our extrapolated melting temperatures can be considered at least as upper bounds. Nevertheless, we have shown that it becomes harder and harder to decrease the melting temperatures with new contributions, so that we still consider that, within uncertainties, our results provide very good estimates of the melting temperatures.

In this work we have also performed a detailed study of uncertainties, which are predominantly of two kinds: on the one hand, we have approximated the effect of heavier hadrons by adding them to the gas as free components. The biggest uncertainty comes from the estimated dependence of their masses on the nonstrange quark mass. On the other hand, we have large uncertainties from the chiral parameters of ChPT and HBChPT. This is the dominant source of error. All in all, our extrapolated melting temperatures come out with an error of the order of $\pm 10 \mathrm{MeV}$, somewhat smaller at $\mu_{B}=0$ and somewhat larger as $\mu_{B}$ increases.

Let us remark that our results are consistent with other $\mu_{B}=0$ model independent results that use ChPT and calculate the partition function either analytically [14] or also with the virial expansion [14-16]. We also find agreement within errors with hadronic models [34], but our melting temperatures come out systematically higher than estimates using lattice $[4,32]$. In addition, our melting curves lie, as expected, above the chemical freeze-out temperature that has been estimated from $p_{\perp}$ spectra at RHIC [29].

Finally, in order to have a more realistic description of the high momentum part of the virial integrals, we have used a unitarized version of ChPT that extends its applicability up to $\sim 1.2 \mathrm{GeV}$, and of HBChPT that generates the
$\Delta(1232)$ resonance. It also allows one to describe correctly the decay widths of the lightest resonances, which are the most abundant of the "heavier hadrons" that we had approximated as free components. We have found that our results are rather stable, within uncertainties, to these changes at $\mu_{B}=0$, but that these effects could produce a further decrease of the melting temperatures of $\sim 10 \mathrm{MeV}$ for higher chemical potentials.

In conclusion, we have presented a model independent and systematic study of the chiral condensate evolution with temperature and chemical potential, by means of the virial expansion and chiral perturbation theory. The highlights of this approach with respect to other fundamental approaches like QCD on the lattice are: the use of physical masses for the hadrons, the good control of the hadron mass dependence on quark masses, and the simple implementation of the baryon chemical potential. We hope that our results could serve as a guideline for future works in hadronic models and also on the lattice, in order to understand the phase diagram of QCD.

\section{ACKNOWLEDGMENTS}

Research partially funded by Spanish CICYT Contract No. FPA2005-02327, as well as Banco Santander/ Complutense Contract No. PR27/05-13955-BSCH. J.R.P. research is also partially funded by CICYT Contract No. BFM2003-00856 and is part of the EU integrated infrastructure initiative HADRONPHYSICS PROJECT, under Contract No. RII3-CT-2004-506078.

Note added. - After submitting to publication this work, we became aware of a recent lattice article [38], contested in [39], where they obtain at $\mu_{B}=0$, a $T_{c}=192(7) \times$ (4) $\mathrm{MeV}$, somewhat higher than all other lattice results we are aware of, and in fairly good agreement with the ChPT results [14-16] and here.
[1] E. B. Gregory, S. H. Guo, H. Kroger, and X. Q. Luo, Phys. Rev. D 62, 054508 (2000); Z. Fodor and S. D. Katz, J. High Energy Phys. 03 (2002) 014; C. R. Allton et al., Phys. Rev. D 66, 074507 (2002); P. de Forcrand and O. Philipsen, Nucl. Phys. B673, 170 (2003).

[2] P. de Forcrand and O. Philipsen, Nucl. Phys. B642, 290 (2002).

[3] C. R. Allton et al., Phys. Rev. D 68, 014507 (2003).

[4] Z. Fodor and S. D. Katz, J. High Energy Phys. 04 (2004) 050 .

[5] S. Muroya, A. Nakamura, C. Nonaka, and T. Takaishi, Prog. Theor. Phys. 110, 615 (2003).

[6] S. Weinberg, Physica A (Amsterdam) 96, 327 (1979).
[7] J. Gasser and H. Leutwyler, Ann. Phys. (Berlin) 158, 142 (1984).

[8] J. Gasser and H. Leutwyler, Nucl. Phys. B250, 465 (1985); B250, 517 (1985); B250, 539 (1985).

[9] A. Dobado, A. Gómez Nicola, A. L. Maroto, and J. R. Peláez, Effective Lagrangians for the Standard Model, Texts and Monographs in Physics (Springer-Verlag, Berlin, 1997); A. Pich, Rep. Prog. Phys. 58, 563 (1995); U. G. Meissner, Rep. Prog. Phys. 56, 903 (1993).

[10] E. Jenkins and A. V. Manohar, Phys. Lett. B 255, 558 (1991); V. Bernard, N. Kaiser, and U. G. Meissner, Int. J. Mod. Phys. E 4, 193 (1995).

[11] G. M. Welke, R. Venugopalan, and M. Prakash, Phys. Lett. 
B 245, 137 (1990); V.L. Eletsky, J. I. Kapusta, and R. Venugopalan, Phys. Rev. D 48, 4398 (1993).

[12] R. Venugopalan and M. Prakash, Nucl. Phys. A546, 718 (1992).

[13] R. Dashen, S. Ma, and H. J. Bernstein, Phys. Rev. 187, 345 (1969).

[14] P. Gerber and H. Leutwyler, Nucl. Phys. B321, 387 (1989).

[15] A. Dobado and J.R. Pelaez, Phys. Rev. D 59, 034004 (1999).

[16] J. R. Pelaez, Phys. Rev. D 66, 096007 (2002).

[17] J. I. Kapusta, Finite-temperature Field Theory (Cambridge University Press, Cambridge, England, 1989).

[18] M. Jamin, Phys. Lett. B 538, 71 (2002).

[19] J. Bijnens, G. Colangelo, and J. Gasser, Nucl. Phys. B427, 427 (1994).

[20] A. Gomez Nicola and J. R. Pelaez, Phys. Rev. D 65, 054009 (2002).

[21] M. Mojzis, Eur. Phys. J. C 2, 181 (1998); G. Ecker and M. Mojzis, Phys. Lett. B 365, 312 (1996).

[22] A. Gomez Nicola, J. Nieves, J. R. Pelaez, and E. Ruiz Arriola, Phys. Lett. B 486, 77 (2000); Phys. Rev. D 69, 076007 (2004).

[23] N. Fettes, U. G. Meissner, and S. Steininger, Nucl. Phys. A640, 199 (1998).

[24] A. Gomez Nicola and J.R. Pelaez, Phys. Rev. D 62, 017502 (2000).
[25] N. Fettes and U.G. Meissner, Nucl. Phys. A676, 311 (2000).

[26] U. G. Meissner, Proc. Sci., LAT2005 (2006) 009 [hep-lat/ 0509029].

[27] T. Becher and H. Leutwyler, Eur. Phys. J. C 9, 643 (1999).

[28] M. Lutz, B. Friman, and C. Appel, Phys. Lett. B 474, 7 (2000).

[29] W. Broniowski and W. Florkowski, Phys. Rev. Lett. 87, 272302 (2001); Acta Phys. Pol. B 33, 1935 (2002).

[30] A. Nyffeler, Z. Phys. C 60, 159 (1993).

[31] A. Tawfik and D. Toublan, Phys. Lett. B 623, 48 (2005).

[32] A. Tawfik, J. Phys. G 31, S1105 (2005).

[33] S. Steininger, U.G. Meissner, and N. Fettes, J. High Energy Phys. 09 (1998) 008.

[34] D. Toublan and J. B. Kogut, Phys. Lett. B 605, 129 (2005).

[35] T. N. Truong, Phys. Rev. Lett. 61, 2526 (1988); A. Dobado, M. J. Herrero, and T. N. Truong, Phys. Lett. B 235, 134 (1990); A. Dobado and J. R. Pelaez, Phys. Rev. D 47, 4883 (1993); 56, 3057 (1997).

[36] J. A. Oller, E. Oset, and J. R. Pelaez, Phys. Rev. Lett. 80, 3452 (1998); Phys. Rev. D 59, 074001 (1999); 60, 099906(E) (1999); F. Guerrero and J. A. Oller, Nucl. Phys. B537, 459 (1999); B602, 641(E) (2001).

[37] J. R. Pelaez, Mod. Phys. Lett. A 19, 2879 (2004).

[38] M. Cheng et al., Phys. Rev. D 74, 054507 (2006).

[39] Y. Aoki, Z. Fodor, S. D. Katz, and K. K. Szabo, hep-lat/ 0609068. 